\title{
Inhibition of the all-trans Retinoic Acid (atRA) Hydroxylases CYP26A1 and CYP26B1 Results in Dynamic, Tissue-Specific Changes in Endogenous atRA Signaling
}

\author{
Faith Stevison, Cathryn Hogarth, Sasmita Tripathy, Travis Kent, and Nina Isoherranen
}

Department of Pharmaceutics, School of Pharmacy, University of Washington, Seattle, Washington (F.S., S.T., N.I.); and School of Molecular Biosciences and the Center for Reproductive Biology, Washington State University, Pullman, Washington (C.H., T.K.)

Received February 1, 2017; accepted April 18, 2017

\begin{abstract}
All-trans retinoic acid (atRA), the active metabolite of vitamin A, is a ligand for several nuclear receptors and acts as a critical regulator of many physiologic processes. The cytochrome P450 family 26 (CYP26) enzymes are responsible for atRA clearance, and are potential drug targets to increase concentrations of endogenous atRA in a tissuespecific manner. Talarozole is a potent inhibitor of CYP26A1 and CYP26B1, and has shown some success in clinical trials. However, it is not known what magnitude of change is needed in tissue atRA concentrations to promote atRA signaling changes. The aim of this study was to quantify the increase in endogenous atRA concentrations necessary to alter atRA signaling in target organs, and to establish the relationship between CYP26 inhibition and altered
\end{abstract}

atRA concentrations in tissues. Following a single $2.5-\mathrm{mg} / \mathrm{kg}$ dose of talarozole to mice, atRA concentrations increased up to 5.7-, 2.7-, and 2.5-fold in serum, liver, and testis, respectively, resulting in induction of Cyp26a1 in the liver and testis and $\operatorname{Rar} \beta$ and Pgc $1 \beta$ in liver. The increase in atRA concentrations was well predicted from talarozole pharmacokinetics and in vitro data of CYP26 inhibition. After multiple doses of talarozole, a significant increase in atRA concentrations was observed in serum but not in liver or testis. This lack of increase in atRA concentrations correlated with an increase in CYP26A1 expression in the liver. The increased atRA concentrations in serum without a change in liver suggest that CYP26B1 in extrahepatic sites plays a key role in regulating systemic atRA exposure.

\section{Introduction}

The active metabolite of vitamin A, all-trans retinoic acid (atRA), is essential in the regulation of many physiologic processes, including embryonic development, immune system function, reproduction, and epithelial integrity, among others (Wolbach and Howe, 1925; Cantorna et al., 1995; Ross, 2012). Due to its many endogenous roles, control of at RA concentrations in various tissues is of critical importance. Decreased levels of RA have been found in a variety of diseases, such as cancer, diabetes, and nonalcoholic fatty liver disease (Moulas et al., 2006; Chang et al., 2008; Liu et al., 2015). In the liver, atRA has been proposed to regulate lipid homeostasis and mitochondrial function via activation of the nuclear retinoic acid receptors (RARs) and peroxisome proliferator activated receptor (PPAR) $\beta / \delta$ (Berry and Noy, 2009; Tripathy et al., 2016). In healthy testes, atRA signaling through RARs has been shown to control sex-specific timing of meiotic initiation and asynchronous spermatogenesis (Griswold, 2016). Importantly, studies in mouse testis containing synchronized germ-cell development have shown that spermatogonial differentiation is associated with a 2- to 5-fold change in intratesticular atRA concentrations (Hogarth et al.,

This work was supported by the National Institutes of Health [Grants R01 GM111772 and T32 GM007750].

https://doi.org/10.1124/dmd.117.075341. 2015a). However, it is not known what magnitude of change in atRA concentrations in different tissues is required to alter atRA signaling.

Maintenance of atRA homeostasis is complex and involves several enzymes. Vitamin A is stored as retinyl esters mainly in the liver, lungs, and adipose, and upon demand, the esters are hydrolyzed to retinol. Retinol is oxidized to atRA via a two-step enzymatic process involving retinol dehydrogenase (RDH) enzymes and the aldehyde dehydrogenase 1A family (ALDH1A) (Napoli, 2012). atRA is eliminated from the body via metabolism to polar metabolites predominantly by the cytochrome P450 26 (CYP26) enzymes CYP26A1 and CYP26B1 (Lutz et al., 2009; Thatcher et al., 2010; Thatcher and Isoherranen, 2009). CYP26A1 and CYP26B1 appear to have functional redundancy as atRA hydroxylases, but have distinct tissue- and cell-type-specific expression (Topletz et al., 2012). For example, CYP26A1 is the predominant CYP26 enzyme in adult human and mouse liver, whereas CYP26B1 protein is not detectable in the liver (Thatcher et al., 2010; Peng et al., 2012). Similarly, cell-type-specific knockouts of CYP26B1 have a distinct phenotype (Li et al., 2009; Hogarth et al., 2015b). Both CYP26A1 and CYP26B1 are expressed in the adult testis and play a role in the onset and maintenance of spermatogenesis (Hogarth et al., 2015a). However, it is not known what effect CYP26 inhibition may have on tissue-specific retinoid signaling. In humans, several variant alleles of CYP26A1 and CYP26B1 have been associated with increased risk of oral cancer, increased size in atherosclerotic lesions, or decreased risk of Crohn's

ABBREVIATIONS: Actb, $\beta$-actin; ALDH1A, aldehyde dehydrogenase 1A family; atRA, all-trans retinoic acid; BID, twice a day; CYP, cytochrome P450; Gusb, $\beta$-glucuronidase; IHC, immunohistochemistry; LC-MS/MS, liquid chromatography-tandem mass spectrometry; NRF1, nuclear respiratory factor 1; PEG 300, polyethylene glycol 300; PGC, peroxisome proliferator activated receptor gamma coactivator; PPAR, peroxisome proliferator activated receptor; q-rtPCR, quantitative reverse-transcriptase polymerase chain reaction; RAR, retinoic acid receptor; RDH, retinol dehydrogenase; SHP, small heterodimer partner; STRA8, stimulated by retinoic acid 8 . 
disease, but at present, it is not known whether these variants alter atRA concentrations or signaling in target tissues (Krivospitskaya et al., 2012; Fransén et al., 2013; Wu et al., 2015).

CYP26 enzymes appear to play a critical role in the control of tissue atRA concentrations, and their expression is believed to be regulated via an autofeedback of atRA concentrations. When atRA levels are increased, such as in pharmacologic dosing of atRA, atRA clearance is significantly increased likely via induction of CYP26 expression, leading to atRA therapy resistance and relapse (Ozpolat et al., 2002; Ross et al., 2011). Upregulation of CYP26 has also been noted in many cancers, suggesting potential changes in atRA concentrations (Shelton et al., 2006; Brown et al., 2014; Wu et al., 2015). Overall, the changes in CYP26 expression suggest that altered atRA signaling contributes to disease development and progression. However, whether endogenous at RA concentrations are altered as a result of or lead to increased CYP26 expression is not known. Inhibition of CYP26s has been proposed as an attractive drug target both to increase endogenous atRA concentrations and to prevent atRA therapy resistance. However, at present, it is not known what magnitude of change in endogenous atRA concentrations is needed to alter atRA signaling in target tissues. In addition, it is not known whether inhibition of CYP26 enzymes increases atRA concentrations in vivo in a manner that can be predicted from in vitro data. The aim of this study was to quantify the increase in endogenous atRA in serum, liver, and testis after administration of the pan-CYP26 inhibitor talarozole and to correlate concentration changes to atRA signaling changes via the RAR and PPAR nuclear receptors in these tissues.

\section{Materials and Methods}

Chemicals and Reagents. All-trans retinoic acid, itraconazole, and mass spectrometry-grade formic acid were purchased from Sigma-Aldrich (St. Louis, MO). $a t$ RA- $_{5}$ was obtained from Santa Cruz Biotechnology (Santa Cruz, CA). Talarozole was purchased from Active Biochem (Maplewood, NJ). Hexanes, water, acetonitrile, and methanol were Optima LC/MS grade from Thermo Fisher Scientific (Waltham, MA). Ethanol, xylene, and Histoplast paraffin used in preparation of slides for immunohistochemistry were from Thermo Fisher Scientific, and HARLECO Bouin fixative fluid was from EMD Millipore (Darmstadt, Germany).

Animal Care and Talarozole Treatments. All animal experiments were approved by the Washington State University Animal Care and Use Committee. Male C57BL/6X129 mice were housed in a temperature- and humidity-controlled environment, and food and water were available ad libitum. Two separate talarozole treatment studies were conducted. In the first study, male mice $(n=24)$ were treated with oral $2.5 \mathrm{mg} / \mathrm{kg}$ talarozole dissolved in polyethylene glycol 300 (PEG 300; Sigma-Aldrich) or PEG 300 alone as a vehicle control $(n=3)$. Talarozole-treated mice ( $n=3$ per time point) were euthanized via $\mathrm{CO}_{2}$ asphyxiation followed by cervical dislocation at $0.25,0.5,1,2,4,8,12$, and 24 hours after the single dose of talarozole, whereas vehicle controls were euthanized at 24 hours. In the second study, male mice $(n=5)$ were treated with oral $2.5 \mathrm{mg} / \mathrm{kg}$ talarozole dissolved in PEG 300 twice daily for 3 days or with PEG 300 alone as a vehicle control $(n=5)$. Talarozole-treated mice were fasted overnight and euthanized 4 hours following a final dose of talarozole in the morning of the fourth day of treatment. Vehicle control mice $(n=5)$ were similarly fasted and euthanized at the same time to serve as a baseline control. In both studies, the mice were exsanguinated, and all of the blood was collected into 1.5-ml Eppendorf tubes and serum isolated from blood by centrifugation at $1,100 \times g$ for 10 minutes at $4^{\circ} \mathrm{C}$. Liver and testis were collected for atRA concentration measurements and for RNA analysis. The residual blood content in the tissues was estimated to be $<10 \%$. All samples were collected in a lightprotected environment, snap frozen on dry ice, and stored at $-80^{\circ} \mathrm{C}$ until use. In the multiple-dose study, one testis from each of three mice was processed to be analyzed via immunohistochemistry (IHC). For IHC analysis, testes were fixed in HARLECO Bouin fixative fluid for 5 hours, dehydrated through a graded ethanol series, washed with xylene, embedded in Histoplast LP paraffin wax, and sectioned (4- $\mu \mathrm{m}$ sections) onto charged glass slides.
Determination of Talarozole Pharmacokinetic Parameters in the Mouse. To prepare mouse serum samples for analysis of talarozole by liquid chromatography-tandem mass spectrometry (LC-MS/MS), $80 \mu 1$ of 1:3 methanol: acetonitrile with $10 \mathrm{nM}$ itraconazole as the internal standard was added to $40 \mu \mathrm{l}$ of serum, the samples were centrifuged at $3000 \times g$ for 20 minutes at $4^{\circ} \mathrm{C}$, and supernatant was collected for quantification. Concentrations of talarozole and internal standard were measured using an AB Sciex (Framingham, MA) API 4500 triple quadrupole mass spectrometer. Analytes were separated using a $100 \times$ 2.1-mm, 1.9- $\mu \mathrm{m}$ C18 Hypersil Gold column (Thermo Fisher Scientific) coupled to a Shimadzu (Kyoto, Japan) LC-20AD liquid chromatography system. Ten microliters of sample was injected, and the mobile phase flow was $500 \mu \mathrm{l} / \mathrm{min}$. LC solvents were A: $0.1 \%$ formic acid in water, and B: acetonitrile. The following gradient was used: $0 \rightarrow 0.5$ minutes $5 \% \mathrm{~B}, 0.5 \rightarrow 3.5$ minutes gradient to $90 \% \mathrm{~B}, 3.5 \rightarrow 5$ minutes $90 \% \mathrm{~B}$, then returned to initial conditions over 0.1 minutes and equilibrated at 5\% B until 7 minutes. Analytes were monitored using positive ion electrospray ionization and $\mathrm{m} / \mathrm{z}$ transitions of $378.1 \rightarrow 239.0$ for talarozole and $705.2 \rightarrow 392.2$ for itraconazole. For mass spectrometry parameters, a declustering potential of 16 , collision energy of 33, and collision cell exit potential of 4 for talarozole and declustering potential of 76 , collision energy of 43 , and collision cell exit potential of 6 for itraconazole were used, and the source temperature was $450^{\circ} \mathrm{C}$. Data were analyzed using Analyst software (AB Sciex, Foster City, CA) and talarozole concentrations were quantified with a standard curve ranging from 0.5 to $50 \mathrm{nM}$.

A one-compartment model with first-order absorption and elimination (eq. 1) was fit to data collected from the single $2.5-\mathrm{mg} / \mathrm{kg}$ dose of talarozole in Phoenix WinNonlin v6.3 (Pharsight, St. Louis, MO) using sparse sampling fitting methods:

$$
C=\frac{F \times D \times k_{a}}{V\left(k_{a}-k\right)} \times\left(e^{-k(t)}-e^{-k_{a}(t)}\right)
$$

where $F$ is bioavailability, $D$ is the dose, $k_{a}$ is the rate of absorption, $k$ is the rate of elimination, and $V$ the volume of distribution of talarozole.

Talarozole Protein Binding. The unbound fraction of talarozole in mouse serum was determined by ultracentrifugation as previously described (Shirasaka et al., 2013). Serum from mice treated with oral $2.5 \mathrm{mg} / \mathrm{kg}$ talarozole as a single dose or twice daily for 3 days was pooled into three groups ( $n=7$ per group), which was then aliquoted into ultracentrifuge tubes. Samples $(n=2$ per group) were incubated for 90 minutes at $37^{\circ} \mathrm{C}$ or centrifuged at $435,000 \times g$ at $37^{\circ} \mathrm{C}$ for 90 minutes in a Sorvall Discovery M150 SE ultracentrifuge (Thermo Fisher Scientific, Waltham, MA) with a Thermo Fisher Scientific S100-AT3 rotor. Supernatant or incubated sample $(40 \mu \mathrm{l})$ was added to $80 \mu \mathrm{l}$ of $1: 3$ methanol:acetonitrile with $10 \mathrm{nM}$ itraconazole as the internal standard and centrifuged at $3,000 \times g$ at $4^{\circ} \mathrm{C}$ for 20 minutes. The supernatant was transferred, and talarozole concentrations were analyzed by LC-MS/MS as described earlier. For each group, the fraction unbound was calculated as the ratio between mean talarozole concentrations with (unbound concentration) and without (total concentration) ultracentrifugation. Final fraction unbound is reported as the mean \pm standard deviation of the three groups.

Prediction of CYP26 Inhibition following Single or Multiple Doses of Talarozole. The change in CYP26A1 and CYP26B1 activity following a single dose of talarozole was predicted according to eq. 2 :

$$
\text { CYP26 activity remaining }(\%)=100 \times \frac{1}{\left(1+\frac{I_{u}}{K_{i}}\right)}
$$

where $I_{u}$ is the unbound talarozole concentration, and $K_{i}$ is the reported inhibition constant for human CYP26A1 or CYP26B1 (Diaz et al., 2016). Based on the high sequence similarity and conserved function as retinoic acid hydroxylases, the human and mouse CYP26 enzymes are not believed to be functionally different from each other (Thatcher and Isoherranen, 2009).

Induction of CYP26A1 protein in the liver was incorporated to predict the change in CYP26A1 activity following multiple doses of talarozole using eq. 3 :

$$
\text { CYP26 activity remaining }(\%)=100 \times \frac{1}{\left(1+\frac{I_{u}}{K_{i}}\right)} * E_{\text {ind }}
$$

where $E_{\text {ind }}$ is the observed fold increase in CYP26A1 protein in the liver measured by Western blot following the final dose of $2.5 \mathrm{mg} / \mathrm{kg}$ talarozole administered twice daily for 3 days. 

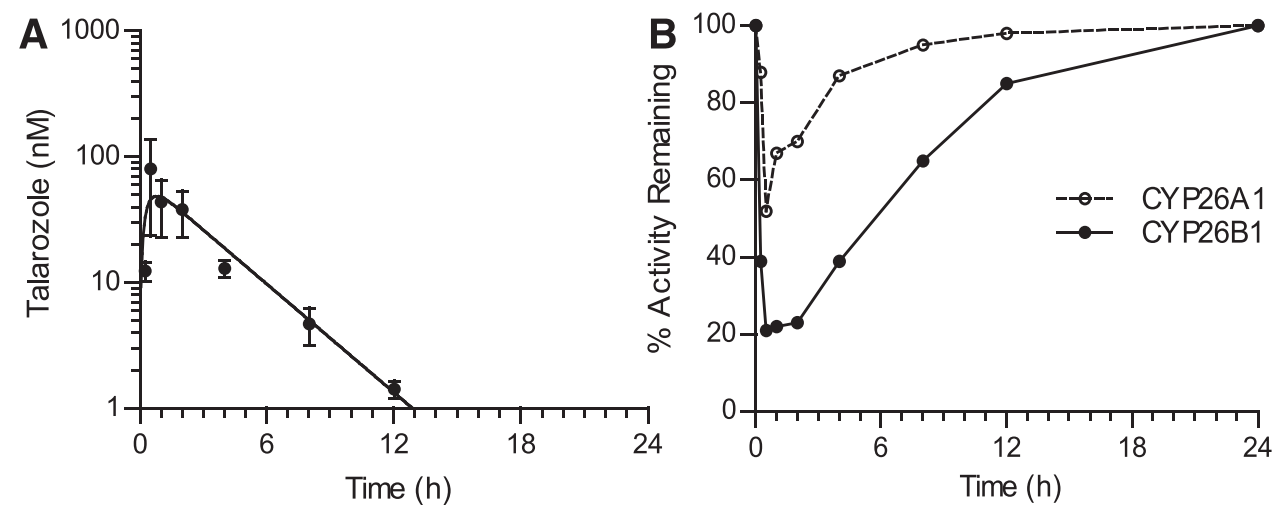

Fig. 1. Talarozole pharmacokinetics and predicted inhibition of CYP26A1 and CYP26B1 by talarozole. (A) Serum concentration versus time profile of talarozole after oral dosing of $2.5 \mathrm{mg} / \mathrm{kg}$ to mice. Data are shown as the mean \pm S.D. $(n=3)$. (B) Predicted change in CYP26A1 and CYP26B1 activity as a function of time following a single dose of $2.5 \mathrm{mg} / \mathrm{kg}$ talarozole to mice.
Quantitation of atRA in Mouse Serum and Tissues. atRA was quantified in serum, liver, and testis as previously described (Arnold et al., 2015a,b). In brief, liver (68-84 mg) or testis (65-126 mg) tissue samples were homogenized with a 1 : 1 volume of saline, and $10 \mu \mathrm{l}$ of $1.5 \mu \mathrm{M}$ atRA-d $\mathrm{d}_{5}$ as the internal standard was added. Each homogenate or serum sample (50 $\mu \mathrm{l}$ of serum plus $10 \mu \mathrm{l}$ of $1.5 \mu \mathrm{M}$ $a t$ RA- $\mathrm{d}_{5}$ ) was transferred to a $15-\mathrm{ml}$ glass culture tube and extracted in a 2:1 volume of acetonitrile $+1 \%$ formic acid and $10 \mathrm{ml}$ of hexanes. The organic layer was separated by centrifugation, dried under nitrogen flow, and reconstituted in 60:40 acetonitrile:water for LC-MS/MS analysis. All extractions were performed on ice and under red light to ensure atRA stability.

The concentrations of $a t \mathrm{RA}$ with $a t \mathrm{RA}-\mathrm{d}_{5}$ as an internal standard in serum and tissue homogenates were measured using an AB Sciex (Framingham, MA) qTrap 5500 mass spectrometer equipped with an Agilent Technologies (Santa Clara, CA) 1290 Infinity ultrahigh-pressure liquid chromatography system as previously described (Arnold et al., 2012, 2015b). In brief, atRA was separated with an Ascentis Express RP-Amide $15 \mathrm{~cm} \times 2.1 \mathrm{~mm}, 2.7-\mu \mathrm{m}$ column (Sigma-Aldrich) with an Ascentis Express RP-Amide 2.7- $\mu \mathrm{m}$ guard column with a mobile phase flow of $500 \mu \mathrm{l} / \mathrm{min}$ and solvents A (0.1\% formic acid in water) and B (60:40 acetonitrile:methanol plus $0.1 \%$ formic acid). The gradient was $0 \rightarrow 2$ minutes $40 \%$ $\mathrm{B}, 2 \rightarrow 10$ minutes increase to $95 \% \mathrm{~B}, 10 \rightarrow 15$ minutes hold at $95 \% \mathrm{~B}$ before returning to initial conditions. For detection, positive ion mode atmospheric pressure chemical ionization was used and $\mathrm{m} / \mathrm{z}$, transitions of $301.2 \rightarrow 205.3$ and $306.2 \rightarrow 116.0$ were monitored for $a t R A$ and $a t R A-d_{5}$ quantification, respectively. Data were analyzed using Analyst software (AB Sciex, Foster City, CA).

Quantification of mRNA and Protein Changes in Mouse Liver and Testis. Mouse liver and testis mRNA was extracted as previously published (Tripathy et al., 2016). In brief, $1 \mathrm{ml}$ of TRI reagent (Invitrogen, Grand Island, $\mathrm{NY}$ ) was added to approximately $100 \mathrm{mg}$ of liver or testis tissue, and mRNA was extracted according to the manufacturer's recommendations. Total mRNA was quantified using a Nanodrop 2000c spectrophotometer (Thermo Fisher Scientific, Waltham, MA), and cDNA was generated using $1 \mu \mathrm{g}$ of RNA and TaqMan Reverse Transcription Reagents (catalog number N8080243; Applied Biosystems, Carlsbad, CA). Changes in Rdh1, Rdh11, Aldh1a1, Aldh1a2, Cyp26a1, Cyp26b1, Rar $\beta$, small heterodimer partner (Shp), peroxisome proliferator activated receptor gamma coactivator $1 \alpha(\operatorname{Pgc} 1 \alpha), \operatorname{Pgc} 1 \beta$, nuclear respiratory factor 1 (Nrf1), and stimulated by retinoic acid 8 (Stra8) mRNA were measured by quantitative reverse-transcriptase polymerase chain reaction (q-rtPCR) using a StepOnePlus (Applied Biosystems) instrument with TaqMan real-time gene expression master mix and PCR primers and probes as described previously (Tripathy et al., 2016). The primer probe pairs were obtained from Applied Biosystems and included Rdh1 (Mm00650636_m1), Rdh11 (Mm00458129_m1), Aldh1a1 (Mm00657317_m1), Aldh1a2 (Mm00501306_m1), Cyp26a1 (Mm00514486_m1), Cyp26b1 (Mm00558507_m1), Rarß (Mm01319677_m1), Shp (Mm0044278_m1), Pgc1 $\alpha$ (Mm01208835_m1), Pgc1 $\beta$ (Mm00504730_m1), Nrf1 (Mm01135606_m1), Stra8 (Mm00486473_m1), $\beta$-glucuronidase (Gusb; Mm00446953_m1), and $\beta$-actin (Actb; Mm00607939_s1). Both Gusb and Actb were evaluated as housekeeping genes for liver and testis, and based on the data, Gusb was used as a housekeeping gene in the liver and Actb in the testis. All samples were analyzed in duplicate, and any undetermined cycle threshold $\left(\mathrm{C}_{\mathrm{T}}\right)$ values were assigned a $C_{T}$ value of 40 (the maximum number of cycles). Changes in target mRNA were measured using relative quantification (fold difference) and the $\Delta \Delta \mathrm{C}_{\mathrm{T}}$ method.

The amount of CYP26A1 protein in livers from mice treated with either vehicle or $2.5 \mathrm{mg} / \mathrm{kg}$ talarozole twice daily for 3 days was measured by Western blot as described previously (Tripathy et al., 2016). The expression of CYP26A1 protein following a single dose of talarozole was not measured, as previous data suggest a delay of CYP26A1 protein synthesis following mRNA induction (Topletz et al., 2015). In brief, livers were homogenized and proteins were extracted using wholeliver lysis buffer in the presence of protease (Roche Applied Science, Indianapolis, IN) and phosphatase inhibitors ( $1 \mathrm{mM} \beta$-glycerol phosphate, $2.5 \mathrm{mM}$ Na-pyrophosphate, $1 \mathrm{mM} \mathrm{Na}_{3} \mathrm{VO}_{4}$ ). Protein concentrations were measured by BCA protein assay (Thermo Fisher Scientific, Waltham, MA). Whole-liver protein extracts were separated by SDS-polyacrylamide gel electrophoresis (NuPAGE-Novex 4-12\% polyacrylamide Bis-Tris; Life Science Technologies, Carlsbad, CA) and transferred to nitrocellulose membranes. Blots were incubated with primary antibodies against CYP26A1 and $\beta$-actin overnight at $4{ }^{\circ} \mathrm{C}$, and the next day, blots were washed and incubated with secondary antibody for 1 hour at room temperature. The CYP26A1 antibody was made in house as previously
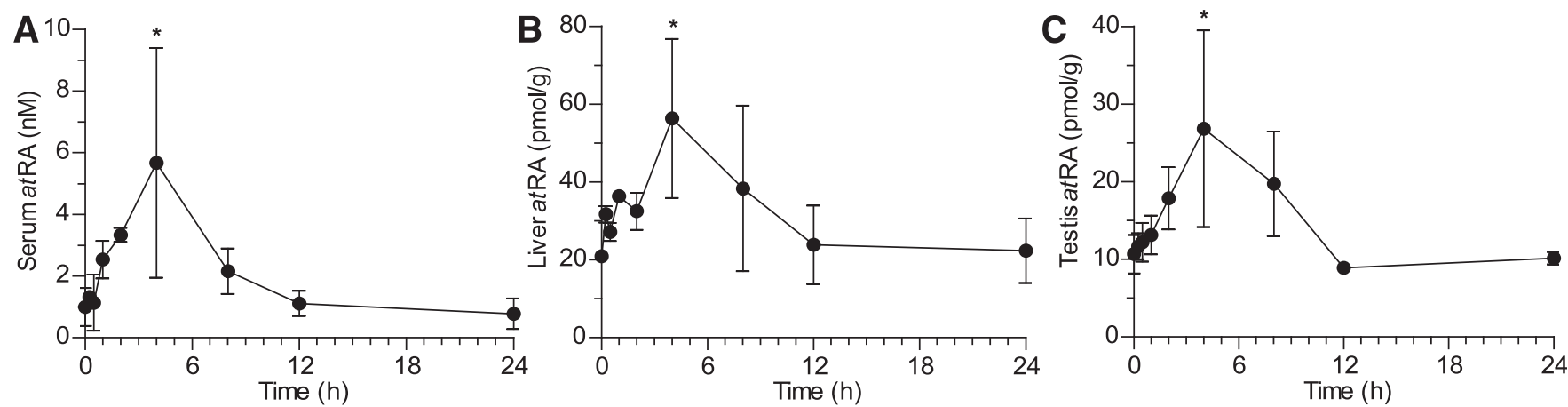

Fig. 2. Changes in the concentrations of endogenous atRA in serum (A), liver (B), and testis (C) as a function of time after a single 2.5 -mg/kg oral dose of talarozole to mice. Data are presented as the mean \pm S.D. $(n=3) . * P<0.05$ compared to control mice. 
A

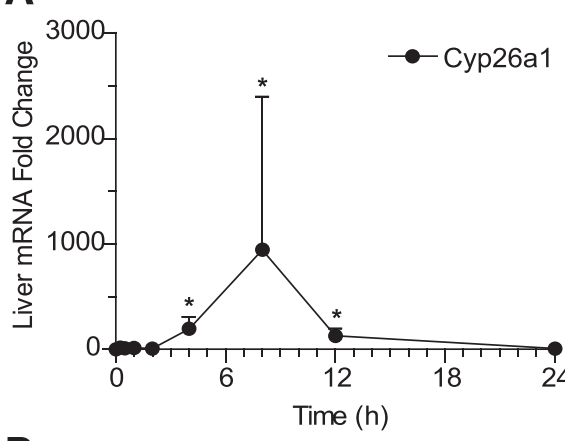

D

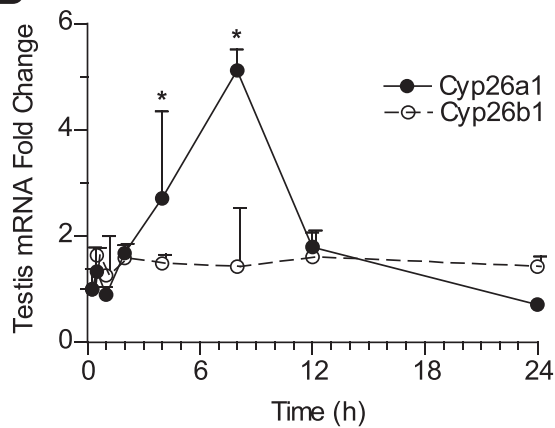

B

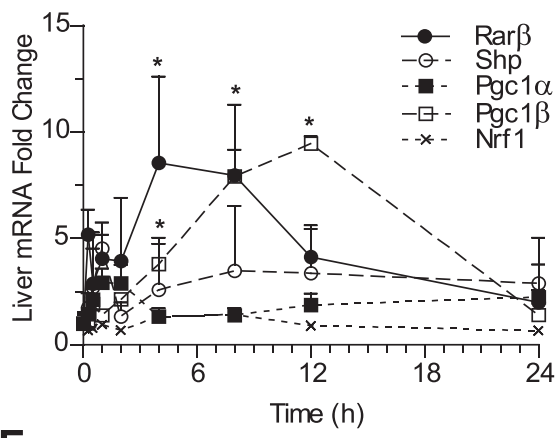

E

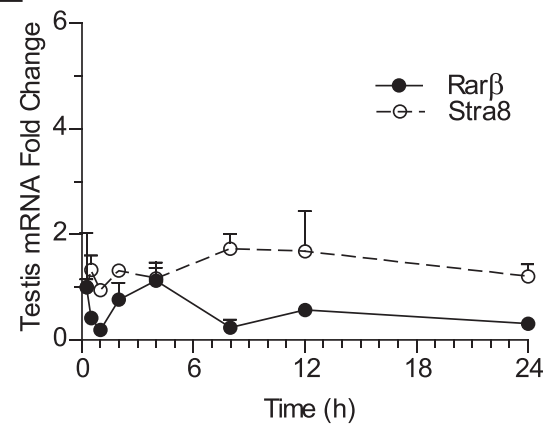

C

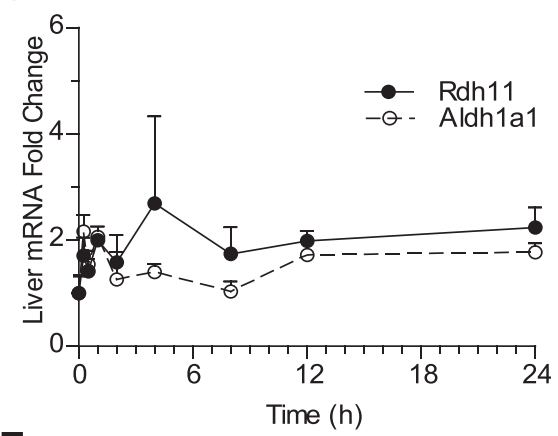

$\mathbf{F}$

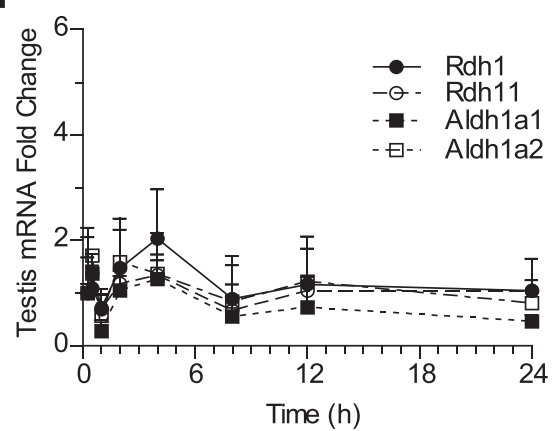

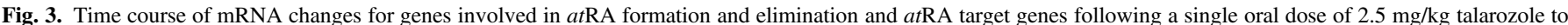

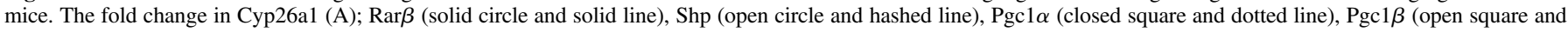

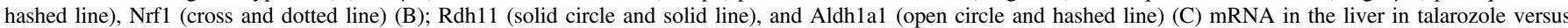

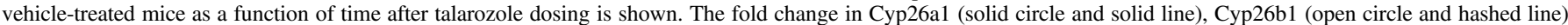

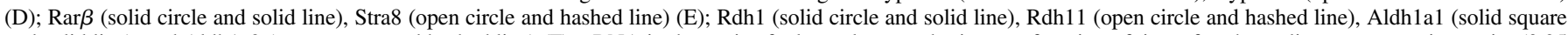

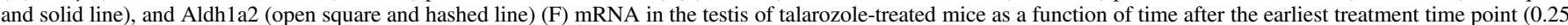
hour) is shown. Data are presented as the mean \pm S.D. $(n=3)$. ${ }^{*} P<0.05$.

described (Thatcher et al., 2010), and the $\beta$-actin antibody (product AC-15) was purchased from Abcam (Cambridge, MA). The secondary antibodies, IRDye 680 (anti-mouse) and IRDye 800 (anti-rabbit), were obtained from LiCor Inc. (Lincoln, NE). Antigen-antibody reactions were detected and quantified using LiCor Odyssey scanner and software (Licor Inc.).

Immunohistochemistry of Stra8 in Mouse Testis. IHC was performed essentially as previously described (Hogarth and Griswold, 2013). In brief, the tissue sections were first immersed in xylene, rehydrated using a graded series of ethanol, and then antigen retrieval was achieved using $0.01 \mathrm{M}$ citrate buffer $(2.94 \mathrm{mg} / \mathrm{ml}$ sodium citrate dihydrate in double distilled water, $\mathrm{pH}$ 6) at a rolling boil for $\sim 5$ minutes. Nonspecific binding of the secondary antibody was blocked via incubation of the tissue sections in a commercially available blocking solution (Histostain Kit, \#956143B; Invitrogen, Carlsbad, CA) for 20 minutes at room temperature. To detect STRA8, sections were incubated at room temperature overnight ( $\sim 16$ hours) in 1:1000 dilution of STRA8 primary antibody in blocking solution. The STRA8 antibody was made in house as described previously (Hogarth et al., 2015a). Control sections were incubated in blocking solution alone. Biotinylated goat-anti-rabbit secondary antibody (Histostain Kit) was applied for 1 hour at room temperature following the manufacturer's instructions. Streptavidin conjugated horseradish peroxidase (Histostain Kit) was then applied for 1 hour at room temperature. Primary antibody binding was visualized by a brown precipitate formed by horseradish peroxidase activity in the presence of 3,3'-diaminobenzidine tetrahydrochloride (Invitrogen, Carlsbad, CA). Tissue sections were then counterstained with a 1:3 dilution Harris Hematoxylin (SigmaAldrich) and dehydrated via a graded ethanol series to xylene. Tissue sections were mounted under glass coverslips using DPX Mounting Media (VWR International, Radnor, PA) or 4',6-diamidino-2-phenylindole-Dihydrochloride (DAPI). Cell types were determined using nuclear morphology and location within the tubules (Russell et al., 1993).

Statistical Analysis. Results are presented as the mean \pm standard deviation. To determine time-dependent changes in atRA tissue concentration or mRNA levels, log-transformed concentration or q-rtPCR data were analyzed by analysis of variance with Dunnett's test as a post-hoc analysis using Prism (GraphPad, La
Jolla, CA). Differences between vehicle control and treated mice in the multipledose study were assessed by $t$-tests on log-transformed data. In all analyses, $P<$ 0.05 was considered significant.

\section{Results}

Effects of Single-Dose Talarozole on CYP26 Activity and Endogenous atRA Concentrations. Following a single $2.5-\mathrm{mg} / \mathrm{kg}$ oral dose of talarozole to mice, the absorption of talarozole was rapid, with a peak concentration ( $80.3 \mathrm{nM}$ ) observed at 30 minutes postdose (Fig. 1). The observed talarozole $\mathrm{AUC}_{0-\infty}$ (area under plasma concentration time curve between time 0 and infinity) was 190 hours*nM, resulting in a relatively high oral clearance of $590 \mathrm{ml} / \mathrm{min} / \mathrm{kg}$. Talarozole had a short half-life in mice (2.2 hours), leading to low to undetectable concentrations of talarozole 12 hours after dosing. The unbound fraction of talarozole in plasma was $5.8 \pm 2.5 \%$.

Based on the reported inhibitory constants for talarozole $(5.1 \mathrm{nM}$ for CYP26A1 and 0.46 nM for CYP26B1) (Diaz et al., 2016), total talarozole concentrations in mice were above the CYP26A1 $K_{i}$ for 8 hours and above the CYP26B $1 K_{i}$ for 12 hours, whereas unbound talarozole concentrations were never above the CYP26A1 $K_{i}$. Using the observed concentrations of talarozole, talarozole unbound fraction, and the $K_{i}$ for each CYP26 isoform, a maximum $48 \%$ decrease in CYP26A1 activity and 79\% decrease in CYP26B1 activity were predicted at 30 minutes postdose (Fig. 1). In agreement with the time above $K_{i}$ and the short half-life of talarozole, at least $80 \%$ of enzyme activity was predicted to return by 4 hours postdose for CYP26A1 and 12 hours postdose for CYP26B1. These results suggest that talarozole is a transient inhibitor of both CYP26 enzymes. 

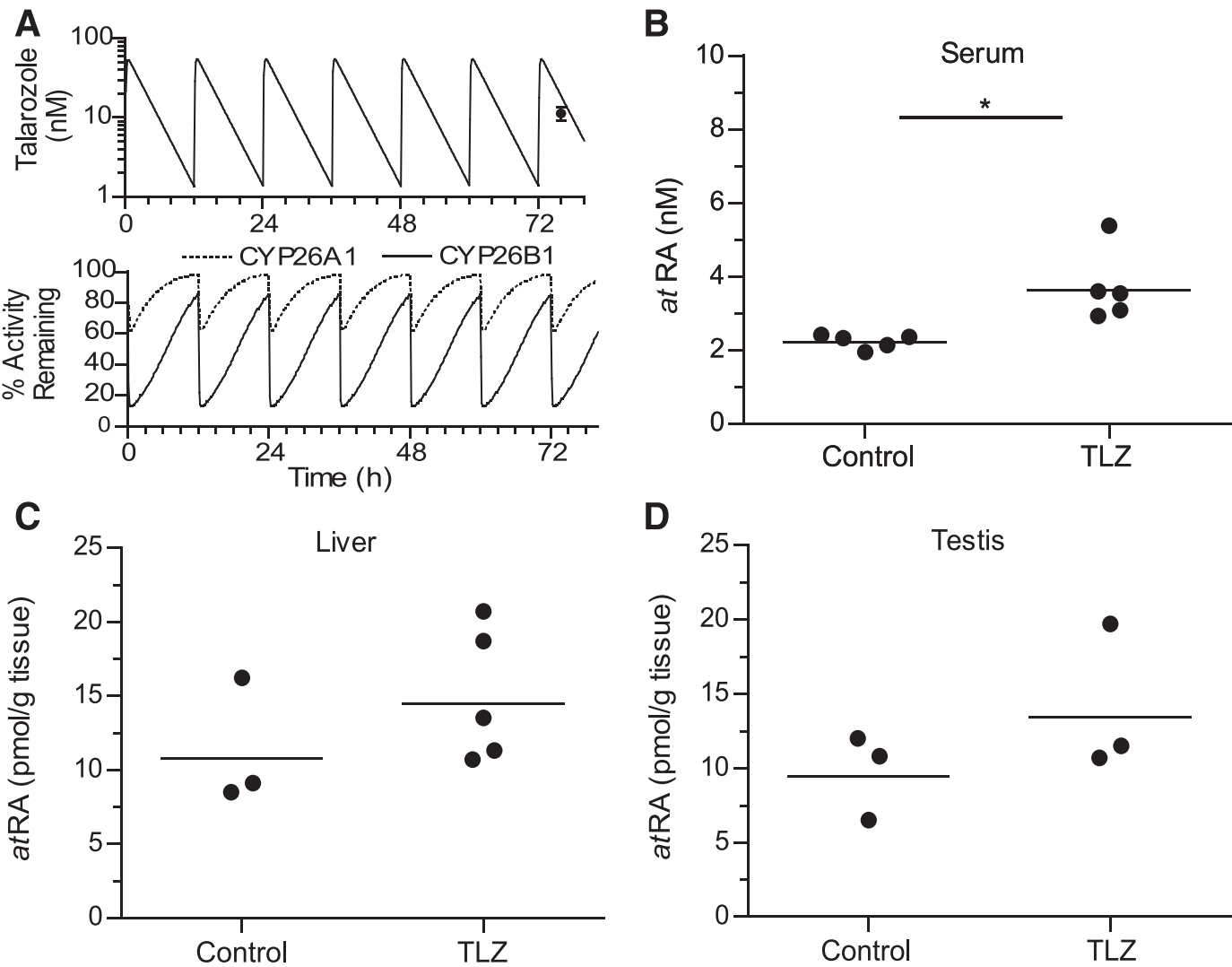

Fig. 4. Predicted change in CYP26 activity and observed concentrations of endogenous atRA following $2.5 \mathrm{mg} / \mathrm{kg}$ talarozole dosed twice a day for 3 days. (A, top) Simulated concentrations of talarozole administered as $2.5 \mathrm{mg} / \mathrm{kg}$ twice a day for 3 days (solid line). A final dose was administered on the fourth day after overnight fast. Observed talarozole concentrations 4 hours following the last $2.5-\mathrm{mg} / \mathrm{kg}$ dose are presented as the mean \pm S.D. $(n=5)$. (A, bottom) Static predictions of CYP26A1 (hashed line) and CYP26B1 (solid line) inhibition following $2.5 \mathrm{mg} / \mathrm{kg}$ talarozole BID for 3 days. Concentrations of atRA in vehicle- and talarozole-treated mice in serum (B), liver (C), and testis (D). Each data point represents an individual animal, and the mean is shown as a solid line. $* P<0.05$. TLZ, talarozole.

Observed serum, liver, and testis concentrations of $a t \mathrm{RA}$ as a function of time after a single dose of talarozole are presented in Fig. 2. Baseline concentrations of atRA in vehicle-treated mice were $1.0 \pm 0.61 \mathrm{nM}$ in serum, $21 \pm 0.48 \mathrm{pmol} / \mathrm{g}$ tissue in liver, and $11 \pm 2.5 \mathrm{pmol} / \mathrm{g}$ tissue in testis. Following talarozole dosing, endogenous atRA concentrations increased transiently with a maximum increase in atRA 4 hours after talarozole dosing. The maximum atRA concentrations were $5.7 \pm 3.7 \mathrm{nM}$, $56 \pm 20 \mathrm{pmol} / \mathrm{g}$ tissue, and $27 \pm 13 \mathrm{pmol} / \mathrm{g}$ tissue in serum, liver, and testis, respectively, representing 5.7-, 2.7-, and 2.5-fold increases over concentrations measured in vehicle treated control mice. In all matrices, concentrations of atRA returned to baseline by 12 hours after the talarozole dose.

To determine whether the 2.5- and 2.7-fold increases in testis and liver atRA concentrations resulted in increased at RA signaling in these organs, changes in the mRNA of Cyp26a1, $\operatorname{Rar} \beta, \operatorname{Shp}, \operatorname{Pgc} 1 \alpha, \operatorname{Pgc} 1 \beta$, and Nrf1 in the liver and Cyp26a1, Cyp26b1, Rar $\beta$, and Stra8 in the testis were measured by q-rtPCR. Cyp26a1 mRNA was increased both in the liver and testis, and similar to $a t$ RA concentrations, the induction was transient and peaked at 8 hours. A significant increase in Cyp26a1 was observed in the liver at 4,8 , and 12 hours (Fig. $3 ; \mathrm{P}<0.05$ ) and at 4 and 8 hours in the testis (Fig. $3 ; \mathrm{P}<0.05$ ). There was no change in Cyp26b1 in the testis (Fig. 3). Of the RAR target genes, $\operatorname{Rar} \beta$ was also significantly increased in the liver at 4 and 8 hours, whereas Shp mRNA was unchanged. In contrast, in the testis, no changes in $\operatorname{Rar} \beta$ or Stra8 mRNA expression were observed (Fig. 3). In the liver, a significant increase in the PPAR $\delta$ target gene $\operatorname{Pgc} 1 \beta$ was observed at $2,4,8$, and 12 hours (Fig. 3; $\mathrm{P}<0.05$ ), whereas $\operatorname{Pgc} 1 \alpha$ and Nrf1 were unchanged.
In addition, no changes were detected over 24 hours following a single dose of talarozole in the mRNA levels of genes that are involved in atRA formation (Rdh11, Aldh1a1, and Aldh1a2) in the liver or testis (Fig. 3). Despite previous reports, no Rdh1 was detected in the liver. Aldh1a2 mRNA was only analyzed in the testis.

Effects of Multiple Doses of Talarozole on CYP26 Activity and Endogenous atRA Concentrations. Multiple doses of talarozole [2.5 $\mathrm{mg} / \mathrm{kg}$ twice a day (BID) for 3 days] were administered to mice to assess the effect of prolonged inhibition of CYP26 on atRA concentrations and signaling. The disposition of talarozole following a single dose was used to simulate the concentrations of talarozole in mice during the 2.5-mg/kg BID dosing for 3 days (Fig. 4). Due to the short half-life of talarozole, no accumulation was expected with multiple dosing. Concentrations of talarozole in serum were measured at 4 hours following the last dose, and the observed concentrations were similar $(11.3 \pm 4.9 \mathrm{nM})$ to those observed at 4 hours in the single-dose study $(12.9 \pm 3.5 \mathrm{nM})$. As in single-dose inhibition predictions, a transient inhibition of CYP26A1 and CYP26B1 was expected during each dosing interval, with the greatest inhibition predicted at 30 minutes post-talarozole dose and complete return of CYP26A1 and CYP26B1 activity by 12 hours following talarozole doses (Fig. 4).

Based on the inhibition data and simulated and observed talarozole concentrations, atRA concentrations were expected to be increased at 4 hours following the last dose of $2.5 \mathrm{mg} / \mathrm{kg}$ talarozole BID administration, as observed following a single dose of talarozole. Indeed, serum at RA concentrations were significantly higher in talarozole-treated compared with vehicle-treated mice (Fig. 4; P $<0.05$ ). However, there 

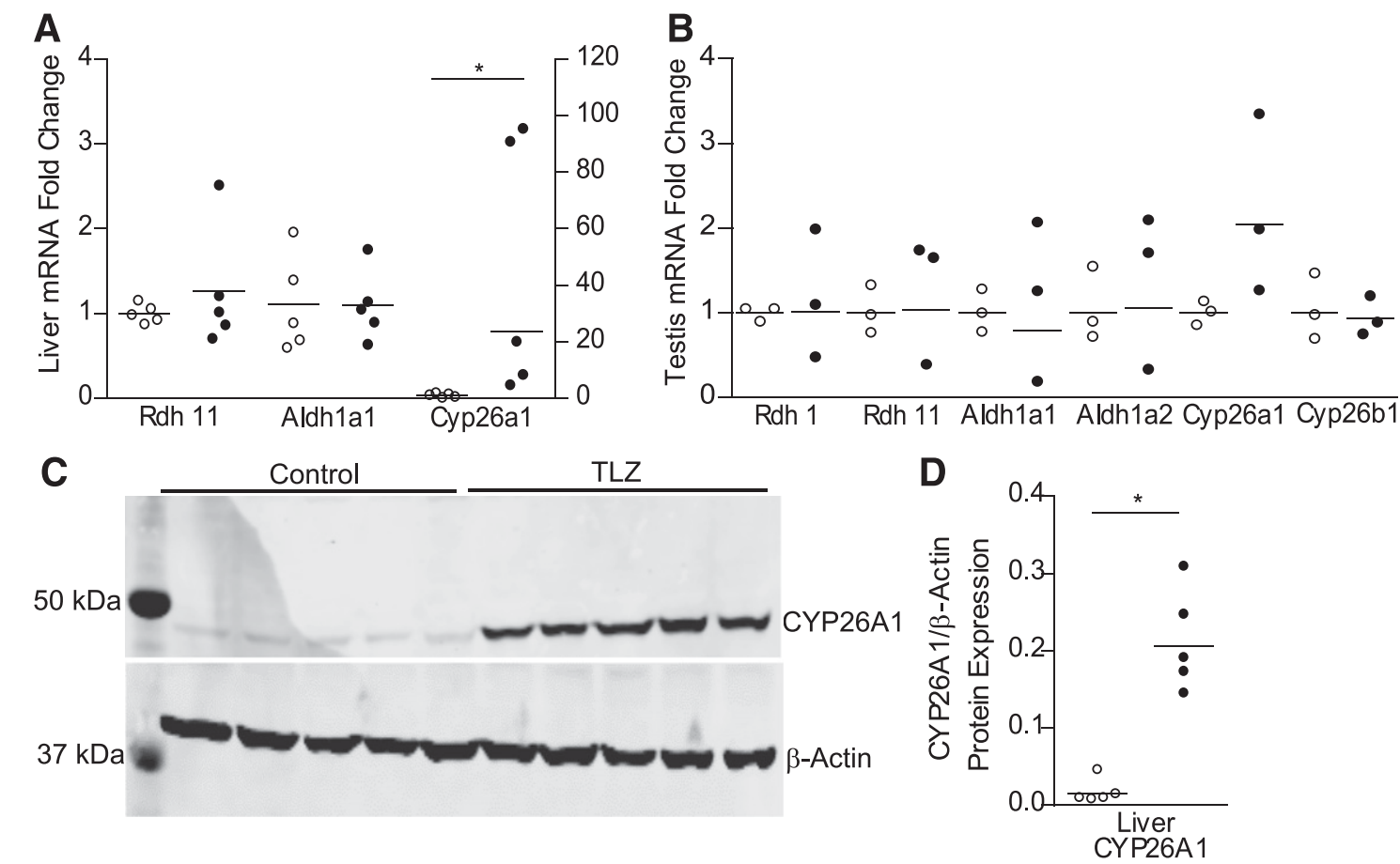

Fig. 5. Fold change in mRNA of enzymes involved in atRA formation and elimination and CYP26A1 protein following dosing of $2.5 \mathrm{mg} / \mathrm{kg}$ talarozole twice a day for 3 days. (A) The fold change in Rdh11 and Aldh1a1 (left axis) and Cyp26a1 (right axis) mRNA in the liver in talarozole- (closed circles) compared with vehicle-treated (open circles) mice. (B) The fold change in Rdh1, Rdh11, Aldh1a1, Aldh1a2, Cyp26a1, and Cyp26b1 mRNA in the testis in talarozole- (closed circles) compared with vehicletreated (open circles) mice. (C) Representative Western blot image of CYP26A1 and $\beta$-actin in vehicle control (lanes 1-5) and talarozole-treated (lanes 6-10) mice. (D) The quantification of signal intensity from Western blot of CYP26A1 protein relative to $\beta$-actin protein in the livers of vehicle control mice (open circles) and mice treated with talarozole (closed circles). Each data point represents an individual animal, and the mean is shown as a solid line. $* P<0.05$.

was no significant increase in endogenous atRA in livers or testes of mice treated with multiple doses of talarozole (Fig. 4). Based on the transient increase in Cyp26a1 mRNA following the single dose of talarozole, and the lack of increase in tissue at RA concentrations despite similar inhibitor exposure as measured after a single dose of talarozole, it was hypothesized that the lack of increase in atRA following multiple doses of talarozole was due to CYP26 induction in target tissues. Therefore, the Cyp26a1 and Cyp26b1 mRNA expression in liver and testis was measured. Cyp26a1 mRNA was significantly higher compared with control mice in livers from talarozole-treated mice (Fig. 5; $\mathrm{P}<0.05$ ). In the testis, a trend of increased Cyp26a1 was observed (Fig. 5), but no significant increase in Cyp26a1 or Cyp26b1 mRNA was observed in testes of mice treated with multiple doses of talarozole.
To determine whether changes in Cyp26a1 mRNA in the liver translated to increased CYP26A1 protein expression, CYP26A1 protein in the liver was measured by Western blot. A mean 11-fold increase in CYP26A1 protein in livers from talarozole-treated versus vehicle-treated mice was measured (Fig. 5). When the 11-fold induction was included together with the transient CYP26A1 inhibition in the prediction of changes in CYP26A1 activity, a net 5-fold increase in CYP26A1 activity in the liver was predicted. Thus, in the liver, which expresses CYP26A1 but not CYP26B1, accounting for transient inhibition as well as induction of CYP26A1 protein predicted a potential decrease in atRA concentrations. To explore potential reasons for the discrepancy between predicted and observed liver $a t$ RA concentrations, the changes in the mRNA of enzymes involved in vitamin A homeostasis (Rdh11 and Aldh1a1 in the liver and Rdh1, Rdh11, Aldh1a1, and Aldh1a2 in the testis) were
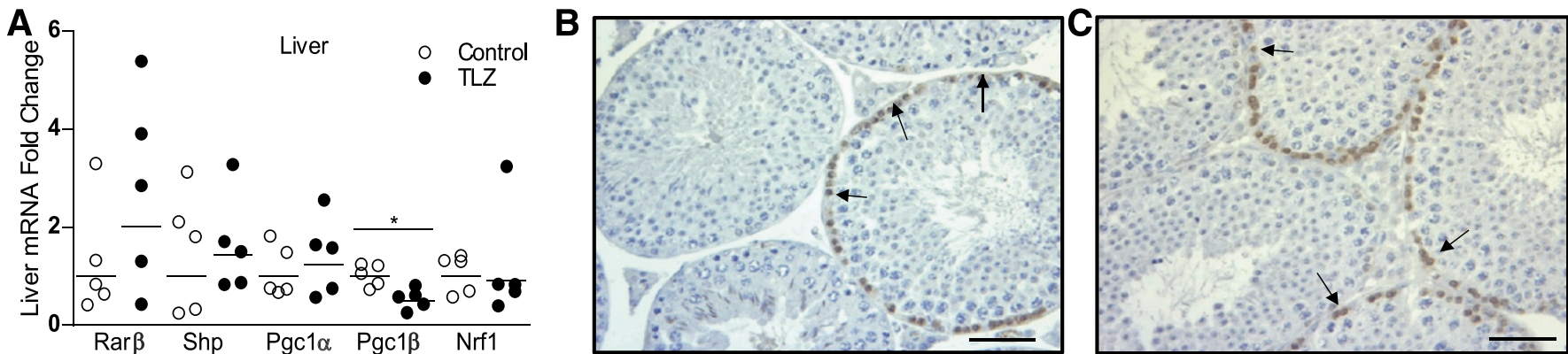

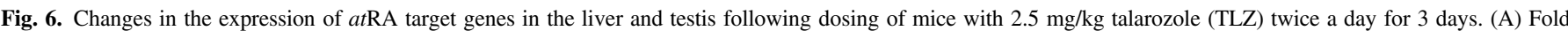

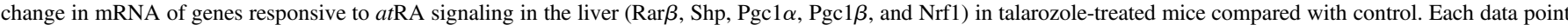

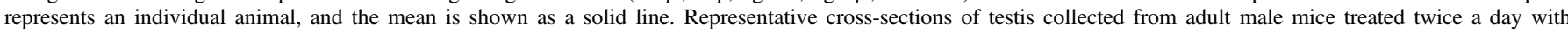

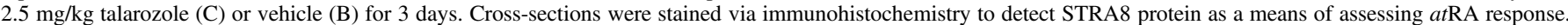
Black arrows indicate STRA8-positive cells. Scale bar $=50 \mu \mathrm{m}$. 
measured. Consistent with results from the single-dose talarozole study, no significant changes in the expression of any of these genes were observed in the liver or testis (Fig. 5).

Effect of CYP26 Inhibition on atRA Signaling in Mouse Liver and Testis. The effect of multiple doses of talarozole on genes that are transcriptionally regulated by atRA in the liver was investigated to further assess atRA signaling. No significant differences were observed in $\operatorname{Rar} \beta$, Shp, $\operatorname{Pgc} 1 \alpha$, or Nrf1 mRNA in the liver (Fig. 6), whereas $\operatorname{Pgc} 1 \beta$ mRNA was decreased in talarozole-treated compared with vehicletreated mice (Fig. 6; $\mathrm{P}<0.05$ ). The impact of CYP26 inhibition on atRA signaling in the testis was assessed by immunohistochemical staining for STRA8, a marker of response to atRA in testicular germ cells (Hogarth et al., 2015a). In agreement with the lack of significant increase in atRA concentrations in the testis, there was no statistically significant increase in STRA8 expression 4 hours following the last dose of multiple doses of talarozole (Fig. 6).

\section{Discussion}

The aim of this study was to determine whether changes in endogenous atRA concentrations will alter retinoid signaling in two target organs, liver and testis, following administration of talarozole, a potent inhibitor of the atRA hydroxylase enzymes CYP26A1 and CYP26B1 according to the model shown in Fig 7. In addition, this study aimed to determine whether the magnitude and time course of changes in the concentrations of an endogenous signaling molecule upon inhibition of its main clearance pathway could be predicted from in vitro data. atRA clearance by CYP26 and the inhibition of CYP26 by talarozole were used as a model system. Inhibition of atRA clearance has been an attractive drug target due to the many tissue-specific roles of atRA and the potential therapeutic benefits of increasing atRA concentrations in various tissues, including the liver and the testis (Njar et al., 2006; Nelson et al., 2013). However, inhibition of atRA clearance could also lead to unwanted side effects due to excessive atRA exposure. As such, a better understanding of the effects of xenobiotic CYP inhibitors on endogenous compound homeostasis is needed to aid in design of more effective inhibitors of key enzymes for therapeutic purposes and in assessing potential toxicity of CYP inhibition.

Talarozole has previously been used as a CYP26 inhibitor to increase atRA levels and signaling in mice, rats, and humans, but no data are available for talarozole disposition in these species (Stoppie et al., 2000; Verfaille et al., 2007a,b). Increased concentrations of atRA have been observed following talarozole dosing, but effects of inhibitor potency and disposition and tissue-specific expression of CYP26 enzymes on atRA concentrations in specific organs have not been reported. This study showed that talarozole dosing caused the greatest changes in serum $a t$ RA concentrations, a finding in good agreement with previously reported data in rats showing the greatest increase in RA concentrations in plasma and skin and the smallest increase in the liver (Stoppie et al., 2000). However, the increase in atRA concentrations in this study was modest in light of the inhibitory potency of talarozole $\left(K_{i}\right.$ $0.46 \mathrm{nM}$ for CYP26B1 and 5.1 nM for CYP26A1) (Diaz et al., 2016). The data presented here suggest that the limiting factor in the efficacy of talarozole is its short half-life, as the inhibition of CYP26 enzymes lasts only a few hours. In addition, the lower potency of talarozole toward CYP26A1 in comparison with CYP26B1 suggests that talarozole will predominantly inhibit CYP26B1 in vivo. As a result, it is likely that talarozole will more effectively increase $a t$ RA concentrations in tissues that have high CYP26B1 expression. This is in agreement with the reported expression of CYP26B1 in the skin and the efficacy of talarozole in disorders of the skin, such as acne and psoriasis (Topletz et al., 2012; Verfaille et al., 2007a,b).

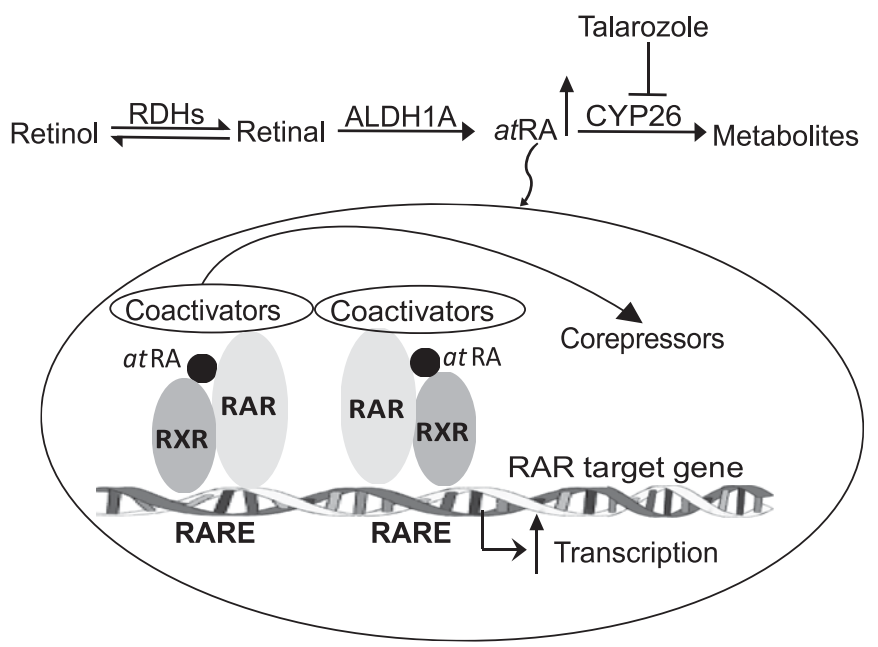

Fig. 7. A proposed schematic of increased RAR-responsive gene transcription in response to CYP26 inhibition by talarozole. Synthesis of atRA from retinol occurs via a two-step enzymatic process involving RDH and ALDH1A enzymes, and CYP26 enzymes are responsible for atRA elimination. Decreased metabolism via CYP26 increases atRA levels, which promotes atRA binding at RAR/RXR heterodimers (Retinoic acid receptor/retinoid $\mathrm{X}$ receptor heterodimers) on retinoic acid response elements (RAREs). Binding of atRA causes dissociation of corepressors to promote gene transcription.

Of the two CYP26 enzymes, CYP26A1 has been shown to be the predominant atRA metabolizing enzyme in the human liver, with CYP26B1 being either absent or below the limit of detection (Thatcher et al., 2010). Hence, altered atRA concentrations in the liver are expected to mainly reflect CYP26A1 inhibition. Both Cyp26a1 and Cyp26b1 mRNA have been detected in mouse testis (Hogarth et al., 2015b), thus inhibition of both enzymes is likely needed to alter intratesticular atRA concentrations significantly. The predicted maximum 50\% decrease in CYP26A1 activity by talarozole after the single dose used in this study is expected to result in an up to 2 -fold increase in atRA concentrations in tissues mainly expressing CYP26A1. The maximum observed 2.7- and 2.5-fold increases in liver and testis atRA concentrations after singledose talarozole are in excellent agreement with this prediction and support a major role of CYP26A1 in regulating liver and testis atRA concentrations. However, the 5.7-fold increase in atRA in serum after a single talarozole dose cannot be explained by inhibition of CYP26A1 and likely reflects inhibition of CYP26B1 in extrahepatic tissues. The predicted $80 \%$ decrease in CYP26B1 activity by talarozole is predicted to result in up to a 5-fold increase in atRA concentrations in tissues mainly expressing CYP26B1. Taken together, these data suggest that circulating atRA levels are largely controlled by CYP26B 1 expression at extrahepatic sites, and indicate that CYP26B1 inhibition by talarozole contributes significantly to the increase in circulating atRA. Although CYP26 inhibition and subsequent increases in at RA concentrations were well predicted after a single dose of talarozole, the lack of any change in liver or testis atRA concentrations following multiple talarozole doses was unexpected. When multiple-dose predictions included CYP26A1 induction, the magnitude of CYP26A1 induction was expected to result in net induction of atRA clearance despite talarozole inhibition and an overall decrease in atRA concentrations in the liver. Yet no decrease in at RA concentrations was observed in the liver. This discrepancy is unlikely to be due to altered atRA synthesis, as Rdh or Aldh1a mRNA was unchanged. The lack of decrease in liver at RA concentrations is more likely due to inhibition of CYP26B1 in extrahepatic tissues (Topletz et al., 2012) and the role of CYP26B1 in regulating RA homeostasis. Serum atRA concentrations, which were elevated despite 
CYP26A1 induction, may contribute significantly to liver atRA concentrations. Thus, to establish whether therapeutic CYP26 inhibition will alter atRA concentrations in target tissues, a better understanding of the relative contributions of individual organs and CYP26A1 and CYP26B1 to atRA clearance is needed. These data also suggest that, in the liver and testis, $>90 \%$ inhibition of CYP26s must be achieved to overcome autoinduction of CYP26A1 to increase atRA concentrations.

The brief inhibition of CYP26A1 and CYP26B1 activity in the singledose study was sufficient to increase atRA concentrations in serum, liver, and testis of mice, although the increase in atRA concentrations was transient. The time course of the observed increase in endogenous atRA following a single dose of talarozole is in good agreement with the previously published atRA half-life ranging from 30 minutes in mouse liver to 1.3 hours in mouse testis (Arnold et al., 2015a). Based on the maximum predicted inhibition of CYP26A1 and CYP26B1 by talarozole at 30 minutes postdose, peak concentrations of atRA would be predicted to occur four atRA half-lives later or between 2.5 and 5.7 hours following talarozole administration. This predicted time course of the peak effect is in good agreement with the observed peak concentrations at 4 hours.

The transient and modest approximately 2-fold increase in at RA concentrations in the liver and testis following a single dose of talarozole was nevertheless sufficient to induce Cyp26a1 in both organs. This demonstrates that even small changes in the concentrations of endogenous signaling molecules can cause major changes in specific signaling pathways. This is also in excellent agreement with the observed 2- to 5 -fold changes in testis atRA concentrations and altered atRA signaling across the spermatogenic cycle (Hogarth et al., 2015a). The rapid time course of change in Cyp26a1 induction suggests that the Cyp26a1 mRNA half-life is only $2-3$ hours in the liver and testis, which is much shorter than the 7-hour half-life determined for Cyp26a1 mRNA in HepG2 cells (Tay et al., 2010). This is biologically plausible as it allows rapid dynamic changes in CYP26 expression in response to environmental factors and atRA exposure (Topletz et al., 2015). Several other genes, including the RAR target gene $\operatorname{Rar} \beta$ and the PPAR $\delta$ target gene $\operatorname{Pgc} 1 \beta$, which have previously been shown to be inducible by atRA, were also responsive to the observed increase in endogenous atRA following a single dose of talarozole. In contrast, Shp, $\operatorname{Pgc} 1 \alpha, \operatorname{Nrf1}$, Cyp26b1, and Stra8, which also have been shown to be inducible by atRA, were not altered by single-dose talarozole treatment in the liver or testis, possibly due to the small change in atRA concentrations. These differences in gene responses are likely due to diversity in inherent responses to atRA and other regulatory elements for these genes. Multiple doses of exogenous atRA have previously been shown to induce Shp mRNA in mouse liver (Koh et al., 2014). Although the magnitude of increase in atRA concentrations was not measured in that study, it is likely to have been much greater than 2-fold, as therapeutic atRA concentrations can exceed endogenous concentrations by 100 fold. Similarly, administration of atRA to mice led to signaling changes in atRA-responsive mitochondrial biogenesis genes $\operatorname{Pgc} 1 \alpha, \operatorname{Pgc} 1 \beta$, and Nrf1 in the liver (Tripathy et al., 2016) and STRA8 in the testis (Hogarth et al., 2015a). Yet in the current study, no effect in these genes was observed following the 3-day dosing of talarozole, likely due to the lack of change in liver and testis atRA concentrations.

In conclusion, inhibition of CYP26A1 and CYP26B1 with a single dose of talarozole led to a modest, transient increase in endogenous atRA and increased atRA-mediated signaling in the liver and testis. However, induction of CYP26A1 upon multiple dosing with talarozole was sufficient to overcome the effect of CYP26A1 inhibition in these tissues. Inhibition of CYP26B1 by talarozole appears to drive the change in atRA concentrations observed in serum, and specific inhibition of CYP26B1 may be sufficient to obtain tissue-specific changes in atRA concentrations.

\section{Authorship Contributions}

Participated in research design: Stevison, Hogarth, Kent, Isoherranen.

Conducted experiments: Stevison, Hogarth, Tripathy, Kent.

Performed data analysis: Stevison, Hogarth, Tripathy, Isoherranen.

Wrote or contributed to the writing of the manuscript: Stevison, Hogarth, Tripathy, Isoherranen.

\section{References}

Arnold SLM, Amory JK, Walsh TJ, and Isoherranen N (2012) A sensitive and specific method for measurement of multiple retinoids in human serum with UHPLC-MS/MS. J Lipid Res 53: 587-598.

Arnold SLM, Kent T, Hogarth CA, Griswold MD, Amory JK, and Isoherranen N (2015a) Pharmacological inhibition of ALDH1A in mice decreases all-trans retinoic acid concentrations in a tissue specific manner. Biochem Pharmacol 95:177-192.

Arnold SLM, Kent T, Hogarth CA, Schlatt S, Prasad B, Haenisch M, Walsh T, Muller CH, Griswold MD, Amory JK, et al. (2015b) Importance of ALDH1A enzymes in determining human testicular retinoic acid concentrations. J Lipid Res 56:342-357.

Berry DC and Noy N (2009) All-trans-retinoic acid represses obesity and insulin resistance by activating both peroxisome proliferation-activated receptor $\beta / \delta$ and retinoic acid receptor. Mol Cell Biol 29:3286-3296.

Brown GT, Cash BG, Blihoghe D, Johansson P, Alnabulsi A, and Murray GI (2014) The expression and prognostic significance of retinoic acid metabolising enzymes in colorectal cancer. PLoS One 9:e90776.

Cantorna MT, Nashold FE, and Hayes CE (1995) Vitamin A deficiency results in a priming environment conducive for Th1 cell development. Eur J Immunol 25:1673-1679.

Chang C-L, Hong E, Lao-Sirieix P, and Fitzgerald RC (2008) A novel role for the retinoic acidcatabolizing enzyme CYP26A1 in Barrett's associated adenocarcinoma. Oncogene 27: 2951-2960.

Diaz P, Huang W, Keyari CM, Buttrick B, Price L, Guilloteau N, Tripathy S, Sperandio VG, Fronczek FR, Astruc-Diaz F, et al. (2016) Development and characterization of novel and selective inhibitors of cytochrome P450 CYP26A1, the human liver retinoic acid hydroxylase. $J$ Med Chem 59:2579-2595.

Fransén K, Franzén P, Magnuson A, Elmabsout AA, Nyhlin N, Wickbom A, Curman B, Törkvist L, D'Amato M, Bohr J, et al. (2013) Polymorphism in the retinoic acid metabolizing enzyme CYP26B1 and the development of Crohn's disease. PLoS One 8:e72739.

Griswold MD (2016) Spermatogenesis: The Commitment to Meiosis. Physiol Rev 96: 1-17.

Hogarth CA, Arnold S, Kent T, Mitchell D, Isoherranen N, and Griswold MD (2015a) Processive pulses of retinoic acid propel asynchronous and continuous murine sperm production. Biol Reprod 92:37.

Hogarth CA, Evans E, Onken J, Kent T, Mitchell D, Petkovich M, and Griswold MD (2015b) CYP26 Enzymes Are Necessary Within the Postnatal Seminiferous Epithelium for Normal Murine Spermatogenesis. Biol Reprod 93:19.

Hogarth CA and Griswold MD (2013) Immunohistochemical approaches for the study of spermatogenesis. Methods Mol Biol 927:309-320.

Koh KH, Pan X, Shen H-W, Arnold SLM, Yu A-M, Gonzalez FJ, Isoherranen N, and Jeong $\mathrm{H}$ (2014) Altered expression of small heterodimer partner governs cytochrome P450 (CYP) 2D6 induction during pregnancy in CYP2D6-humanized mice. J Biol Chem 289: 3105-3113

Krivospitskaya O, Elmabsout AA, Sundman E, Söderström LA, Ovchinnikova O, Gidlöf AC, Scherbak N, Norata GD, Samnegård A, Törmä H, et al. (2012) A CYP26B1 polymorphism enhances retinoic acid catabolism and may aggravate atherosclerosis. Mol Med 18 $712-718$.

Li H, MacLean G, Cameron D, Clagett-Dame M, and Petkovich M (2009) Cyp26b1 expression in murine Sertoli cells is required to maintain male germ cells in an undifferentiated state during embryogenesis. PLoS One 4:e7501.

Liu Y, Chen H, Wang J, Zhou W, Sun R, and Xia M (2015) Association of serum retinoic acid with hepatic steatosis and liver injury in nonalcoholic fatty liver disease. Am J Clin Nutr 102:130-137.

Lutz JD, Dixit V, Yeung CK, Dickmann LJ, Zelter A, Thatcher JE, Nelson WL, and Isoherranen N (2009) Expression and functional characterization of cytochrome P450 26A1, a retinoic acid hydroxylase. Biochem Pharmacol 77:258-268.

Moulas AN, Gerogianni IC, Papadopoulos D, and Gourgoulianis KI (2006) Serum retinoic acid, retinol and retinyl palmitate levels in patients with lung cancer. Respirology 11:169-174.

Napoli JL (2012) Physiological insights into all-trans-retinoic acid biosynthesis. Biochim Biophys Acta 1821:152-167.

Nelson CH, Buttrick BR, and Isoherranen N (2013) Therapeutic potential of the inhibition of the retinoic acid hydroxylases CYP26A1 and CYP26B1 by xenobiotics. Curr Top Med Chem 13 $1402-1428$.

Njar VCO, Gediya L, Purushottamachar P, Chopra P, Vasaitis TS, Khandelwal A, Mehta J, Huynh C, Belosay A, and Patel J (2006) Retinoic acid metabolism blocking agents (RAMBAs) for treatment of cancer and dermatological diseases. Bioorg Med Chem 14:4323-4340.

Ozpolat B, Mehta K, Tari AM, and Lopez-Berestein G (2002) all-trans-Retinoic acid-induced expression and regulation of retinoic acid 4-hydroxylase (CYP26) in human promyelocytic leukemia. Am J Hematol 70:39-47.

Peng L, Yoo B, Gunewardena SS, Lu H, Klaassen CD, and Zhong X-b (2012) RNA sequencing reveals dynamic changes of mRNA abundance of cytochromes P450 and their alternative transcripts during mouse liver development. Drug Metab Dispos 40:1198-1209.

Ross AC (2012) Vitamin A and retinoic acid in T cell-related immunity. Am J Clin Nutr 96: $1166 \mathrm{~S}-1172 \mathrm{~S}$.

Ross AC, Cifelli CJ, Zolfaghari R, and Li N-Q (2011) Multiple cytochrome P-450 genes are concomitantly regulated by vitamin A under steady-state conditions and by retinoic acid during hepatic first-pass metabolism. Physiol Genomics 43:57-67.

Russell LD, Ettlin RA, Hikim APS, and Clegg ED (1993) Histological and histopathological evaluation of the testis. Int $J$ Androl 16:83.

Shelton DN, Sandoval IT, Eisinger A, Chidester S, Ratnayake A, Ireland CM, and Jones DA (2006) Up-regulation of CYP26A1 in adenomatous polyposis coli-deficient vertebrates via a 
WNT-dependent mechanism: implications for intestinal cell differentiation and colon tumor development. Cancer Res 66:7571-7577.

Shirasaka Y, Sager JE, Lutz JD, Davis C, and Isoherranen N (2013) Inhibition of CYP2C19 and CYP3A4 by omeprazole metabolites and their contribution to drug-drug interactions. Drug Metab Dispos 41:1414-1424.

Stoppie P, Borgers M, Borghgraef P, Dillen L, Goossens J, Sanz G, Szel H, Van Hove C, Van Nyen G, Nobels G, et al. (2000) R115866 inhibits all-trans-retinoic acid metabolism and exerts retinoidal effects in rodents. J Pharmacol Exp Ther 293:304-312.

Tay S, Dickmann L, Dixit V, and Isoherranen N (2010) A comparison of the roles of peroxisome proliferator-activated receptor and retinoic acid receptor on CYP26 regulation. Mol Pharmacol 77:218-227.

Thatcher JE and Isoherranen N (2009) The role of CYP26 enzymes in retinoic acid clearance. Expert Opin Drug Metab Toxicol 5:875-886.

Thatcher JE, Zelter A, and Isoherranen N (2010) The relative importance of CYP26A1 in hepatic clearance of all-trans retinoic acid. Biochem Pharmacol 80:903-912.

Topletz AR, Thatcher JE, Zelter A, Lutz JD, Tay S, Nelson WL, and Isoherranen N (2012) Comparison of the function and expression of CYP26A1 and CYP26B1, the two retinoic acid hydroxylases. Biochem Pharmacol 83:149-163.

Topletz AR, Tripathy S, Foti RS, Shimshoni JA, Nelson WL, and Isoherranen N (2015) Induction of CYP26A1 by metabolites of retinoic acid: Evidence that CYP26A1 is an important enzyme in the elimination of active retinoids. Mol Pharmacol 87:430-441.
Tripathy S, Chapman JD, Han CY, Hogarth CA, Arnold SLM, Onken J, Kent T, Goodlett DR, and Isoherranen N (2016) All-trans-retinoic acid enhances mitochondrial function in models of human liver. Mol Pharmacol 89:560-574.

Verfaille CJ, Coel M, Boersma IH, Mertens J, Borgers M, and Roseeuw D (2007a) Oral R115866 in the treatment of moderate to severe facial acne vulgaris: an exploratory study. Br J Dermatol 157:122-126.

Verfaille CJ, Thissen CACB, Bovenschen HJ, Mertens J, Steijlen PM, and van de Kerkhof PCM (2007b) Oral R115866 in the treatment of moderate to severe plaque-type psoriasis. J Eur Acad Dermatol Venereol 21:1038-1046.

Wolbach SB and Howe PR (1925) Tissue changes following deprivation of fat-soluble A vitamin. $J$ Exp Med 42:753-777.

Wu S-J, Chen Y-J, Shieh T-Y, Chen C-M, Wang Y-Y, Lee K-T, Lin Y-M, Chien P-H, and Chen P-H (2015) Association study between novel CYP26 polymorphisms and the risk of betel quid-related malignant oral disorders. ScientificWorldJournal 2015:160185.

Address correspondence to: Dr. Nina Isoherranen, Department of Pharmaceutics, School of Pharmacy, University of Washington, Health Sciences Building Room H-272M, Box 357610, Seattle, WA 98195-7610. E-mail: ni2@u.washington.edu 